\title{
Quasi-Efficient Revocation of Group Signatures
}

\author{
Giuseppe Ateniese ${ }^{1}$, Dawn Song ${ }^{2}$, and Gene Tsudik ${ }^{3}$ \\ ${ }^{1}$ Department of Computer Science \\ The Johns Hopkins University, Baltimore, MD. USA \\ ateniese@cs.jhu.edu \\ 2 Computer Science Division, EECS \\ University of California, Berkeley, CA. USA \\ dawnsong@cs.berkeley.edu \\ 3 Department of Information and Computer Science, \\ University of California, Irvine, CA. USA \\ gts@ics.uci.edu
}

\begin{abstract}
A group signature scheme allows any group member to sign on behalf of the group in an anonymous and unlinkable fashion. In the event of a dispute, a designated trusted entity can reveal the identity of the signer. Group signatures are claimed to have many useful applications such as voting and electronic cash. A number of group signature schemes have been proposed to-date. However, in order for the whole group signature concept to become practical and credible, the problem of secure and efficient group member revocation must be addressed. In this paper, we construct a new revocation method for group signatures based on the signature scheme by Ateniese et al. [ACJT]. This new method represents an advance in the state-of-the-art since the only revocation schemes proposed thus far are either: 1) based on implicit revocation and the use of fixed time periods, or 2) require the signature size to be linear in the number of revoked members. Our method, in contrast, does not rely on time periods, offers constant-length signatures and constant work for the signer.
\end{abstract}

Keywords: Group signatures, revocation of group membership credentials, dynamic groups.

\section{Introduction}

Group signatures are a relatively new concept introduced by Chaum and van Heijst [CvH91] in 1991. A group signature, akin to its traditional counterpart, allows the signer to demonstrate knowledge of a secret with respect to a specific document. A group signature is publicly verifiable: it can be validated by anyone in possession of a group public key. However, group signatures are anonymous in that no one, with the exception of a designated group manager, can determine the identity of the signer. Furthermore, group signatures are unlinkable which makes it computationally hard to establish whether or not multiple signatures are produced by the same group member. In exceptional cases (such as a legal dispute) any group signature can be "opened" by a group manager to reveal unambiguously the identity of the actual signer. At the same time, no one - including the group manager — can misattribute a valid group signature. 
These features of group signatures make them attractive for many specialized applications, such as voting and bidding. They can, for example, be used in invitations to submit tenders [CP95]. All companies submitting a tender form a group and each company signs its tender anonymously using the group signature. Once the preferred tender is selected, the winner can be traced while the other bidders remain anonymous.

More generally, group signatures can be used to conceal organizational structures, e.g,, when a company or a government agency issues a signed statement. Group signatures can also be integrated with an electronic cash system whereby several banks can securely distribute anonymous and untraceable e-cash. The group property can offer a further advantage of concealing the identity of the cash-issuing bank [LR98].

Several interesting group signature schemes have been recently proposed [CP95,CvH91], [CS97,AT99a,CM98a,Cam97]. Some have been subsequently broken, others are impractical due to long public keys and/or long signatures while most remaining schemes offer uncertain (i.e., unproven) security. One exception is a recent group signature scheme by Ateniese et al. [ACJT] which is referred to as ACJT from here on. This scheme is particularly attractive since it is both efficient and provably secure.

Motivation As observed in [AT99], to be truly useful, a group signature scheme must support dynamic group membership. Current state-of-the-art group signature schemes (such as Camenisch/Stadler [CS97], Camenisch/Michels [CM98a], ACJT [ACJT]) support growing membership: new members can join without precipitating changes in the group public key or re-issuing group membership certificates for existing members. However, shrinking group membership has not been given the same attention. We believe this is because either it was not deemed important enough, or (more likely) no viable solutions were known.

In many realistic group settings, group members are equally likely to join, leave voluntarily or be excluded, from the group. Therefore, we consider supporting growing and shrinking membership of equal importance. Starting from this premise, we claim that group signature schemes, no matter how elegant or how secure, will remain a neat and curious tool in a theoretical cryptographer's "bag-of-tricks" until a secure and efficient method to support both growing and shrinking membership is found.

Contribution In this paper, we construct and demonstrate an effective and secure revocation method for group signatures based on the signature scheme by Ateniese et al. [ACJT]. This new method represents an advance in the state-of-the-art since the only revocation schemes proposed thus far are either: ${ }^{1}$

1. Based on implicit revocation, (loosely) synchronized clocks and the use of fixed time periods, or

2. Require group signature size to be $O(n)$ where $n$ is the number of revoked members and ask the signer to perform $O(n)$ work to compute each signature.

Our method, in contrast, offer explicit (CRL-based) revocation, requires no time periods and offers constant-length signatures and constant work for signers.

\footnotetext{
${ }^{1}$ See Section 2 for details.
} 
Broadly speaking, this paper has but one contribution: it demonstrates the first viable group revocation scheme based on the only provably secure and efficient group signature scheme proposed to-date. At the same time, it should be noted from the outset that the revocation method described in this paper - albeit viable - is not quite practical for reasons to be discussed below. However, we believe that this result moves the field one (perhaps, small) step closer to reality.

Organization The rest of the paper is organized as follows. We first provide, in the next section, an overview of related work. In Section 3 we discuss some preliminaries. Next, Section 4 discusses revocation issues in the context of group signatures. Section 5, summarizes the Ateniese et al. group signature scheme. Sections 6 overviews a very simple revocation scheme followed by the new quasi-efficient revocation scheme and its informal analysis presented in Sections 7 and 8, respectively. We conclude the paper with the summary and future work.

\section{Related Work}

Recently, Bresson and Stern [BS2000] have proposed the first solution for revocation of group signatures. Unfortunately, their scheme requires the signature size to be linear with respect to the number of revoked members. Moreover, it is based on the group signature scheme proposed by Camenisch and Stadler which has been found to have certain security problems [AT99], and its corrected/modified version has not been proven secure.

In a very recent paper, Song [Song01] proposed two interesting revocation methods based, like the present work, on the ACJT scheme. (Recall that ACJT is provably secure.) Both methods are notable since - in addition to standard revocation - they also provide retroactive revocation as well as forward security. (In fact, the emphasis is on forward security.) Moreover, they offer constant-length signatures which is an improvement over the Bresson and Stern's result. However, one important feature of Song's methods is the use of fixed (in both length and number) time periods to support revocation. In particular, each member's certificate must evolve in every time period and all verifiers must be aware of this evolution. Also, the maximum number of time periods is fixed and embedded in each member's group certificate. While appropriate for some settings, this solution is not very general since it is hard (in fact, impossible) to revoke a member within a time period. Furthermore, the security of one of the methods is based on a new and uncertain cryptographic assumption which is appreciably stronger than the Decision Diffie-Hellman (DDH) assumption. The second scheme relies on a method (one-way function) of deterministically computing a fixed-length sequence of prime numbers starting with an initial prime which may be inefficient.

\section{Preliminaries}

Group-signature schemes are typically defined as follows: ${ }^{2}$

\footnotetext{
${ }^{2}$ An in-depth discussion on this subject can be found in [Cam98].
} 
Definition 1. A group signature scheme is a digital signature scheme comprised of the following five procedures:

SETUP : A probabilistic algorithm which - on input of a security parameter $\ell$ - outputs the initial group public key $\mathcal{Y}$ (including all system parameters) and the secret key $\mathcal{S}$ for the group manager.

JOIN : A protocol between the group manager and a user that results in the user becoming a new group member. The user's output is a membership certificate and a membership secret.

SIGN : A probabilistic algorithm that on input a group public key, a membership certificate, a membership secret, and a message $m$ outputs group signature of $m$.

VERIFY: An algorithm for establishing the validity of an alleged group signature of a message with respect to a group public key.

OPEN : An algorithm that, given a message, a valid group signature on it, a group public key and a group manager's secret key, determines the identity of the signer.

A secure group signature scheme must satisfy the following properties:

Correctness: Signatures produced by a group member using SIGN must be accepted by VERIFY.

Unforgeability: Only group members are able to sign messages on behalf of the group.

Anonymity: Given a valid signature of some message, identifying the actual signer is computationally hard for everyone but the group manager.

Unlinkability: Deciding whether two different valid signatures were computed by the same group member is computationally hard.

Exculpability: Neither a group member nor the group manager can sign on behalf of other group members. ${ }^{3}$

Traceability: The group manager is always able to open a valid signature and identify the actual signer. Therefore, any colluding subset of group members cannot generate a valid signature that the group manager cannot link to one of the colluding group members.

In order to provide revocation of membership, an additional property is necessary:

Revocability: A signature produced using SIGN by a revoked member must be rejected using a (potentially modified) VERIFY. Equivalently, a signature produced using SIGN by a valid member must be accepted by VERIFY.

The efficiency of of a group signature scheme depends on a number of factors. Usually, the costs of SIGN and VERIFY as well as the sizes of the group signature and the group public key are the most important efficiency measures.

\section{Revocation Preliminaries}

In general, as soon as a member is revoked, there must be a way to unambiguously determine her revocation status. At the same time, it is sometimes desirable that all

\footnotetext{
${ }^{3}$ However, nothing precludes the group manager from creating phantom signers and then producing group signatures. The same risk occurs with respect to CA-s in traditional (non-group) PKI-s.
} 
signatures generated by a group member before revocation remain valid and secure, i.e., anonymous and unlinkable. This property was first defined in [AT99] and later refined and referred by Song [Song01] as backward unlinkability. We observe, however, that a scheme providing backward unlinkability will allow any revoked members to generate signatures in the future and claim that they were generated before revocation occured. This is particularly unpleasant in the context of group signatures where each user signs on behalf of the entire group and signatures are linked to the group's intentions rather than to those of the single signer. Thus, another level of revocation could require to link and identify all the signatures generated by a revoked group member. We refer to this revocation flavor as unconditional linkability.

One simple way to obtain revocation is to issue a new group public key and new group certificates to all valid members whenever a group member (or a number thereof) leaves or is ejected. However, this would entail a heavy cost and a significant inconvenience. First, all potential verifiers must be notified of the change. This appears to be unavoidable. Second, all remaining members must participate in a JOIN protocol with the group manager. This represents an extra burden for the members since the JOIN protocol is always on-line and usually involves significant effort (as compared to SIGN or VERIFY).

However, it is possible to avoid running interactive JOIN protocols with all members. This can be achieved by generating a new group public key and issuing new group certificates for all members without any interaction. As an illustration, we sketch out (in Section 6) a simple method based on the ACJT scheme. (A very similar approach can be constructed with the Camenisch/Stadler group signature scheme [CS97].) However, this approach is not very practical as it involves the issuance of many new membership certificates and requires each group member to fetch its new certificate following every member leave event.

To achieve more effective and efficient revocation, we need to avoid issuing new group certificates to non-revoked members. An ideal revocation method would employ the revocation paradigm commonly used in traditional signature schemes: a verifier simply checks the signer's certificate against the current Certificate Revocation List (CRL). This paradigm is attractive since the signer is unaware of the ever-changing CRL and the revocation checking burden is placed on the verifier. In our setting, however, a group signature always contains in some form an encrypted version of the signer's group certificate. As pointed out in [ACJT], encryption of the certificate must be semantically secure in order to prevent linking of group signatures.

The very same semantic security makes it impossible for the verifier to link a group signature to a (potentially) revoked group certificate (or some function thereof) that has to appear as part of a CRL. To see why this is the case, consider the opposite: if a verifier is able to link a single group signature to a certain CRL entry, then the same verifier can link multiple group signatures (all by one signer) to the very same CRL entry. This is not a problem if the signer is revoked before all of these group signatures are generated. However, if a verifier can link (based on a current CRL) a revoked signer's signatures computed before revocation, the property of backward unlinkability is not preserved. Therefore, we claim that the signer must somehow factor in the current CRL when 
generating a group signature. In fact, the signer must prove, as part of the signing, that its group certificate (or a function thereof) is not part of the current CRL.

The above is the general approach we take in this paper. The method outlined in detail below (in Section 7) requires a signer to prove non-membership of the current CRL as part of signature generation. The verifier, in turn, checks revocation as part of signature verification. The end-result is that the notion of the group public key is extended to include the latest group CRL.

Revocation Efficiency We identify the following measures of efficiency or practicality for any revocation method (of course, only in the context of group signatures):

- Increased Signature Size: is the most important measure of a revocation method's efficiency. Clearly, signature size should be minimized. More generally, if the underlying group signature scheme has $O(x)$-size signatures (where $x$ is a constant, or some function of group size), revocation checking should ideally not change the signature size in the $O()$ notation.

- Signer Cost: is the additional cost of generating a group signature that proves nonrevocation of the signer. Ideally, this added cost is constant.

- Verifier Cost: is the additional cost of verifying a group signature that proves nonrevocation of the signer. As above, this added cost is, at best, constant.

- CRL Size: is an essential measure since it effectively determines the overall size of the group public key.

- CRL Issuance Cost: is the cost of composing and issuing a new CRL (by the group manager) each time a group member must be revoked. While not completely negligible, this efficiency measure is the least significant of the above.

\section{The ACJT Group Signature Scheme}

In this section we provide an overview of the ACJT scheme [ACJT]. (Readers familiar with ACJT may skip this section with no loss of continuity.) In its interactive, identity escrow form, the ACJT scheme is proven secure and coalition-resistant under the Strong RSA and DDH assumptions. The security of the non-interactive group signature scheme relies additionally on the Fiat-Shamir heuristic (also known as the random oracle model).

Let $\Lambda$ and $\Gamma$ be two integral ranges as defined in [ACJT] and let $\mathcal{H}$ be a collisionresistant hash function $\mathcal{H}:\{0,1\}^{*} \rightarrow\{0,1\}^{k}$. The ACJT scheme is defined in the set of quadratic residues $\mathrm{QR}(n)$ where $n=p q$ with $p=2 p^{\prime}+1$ and $q=2 q^{\prime}+1\left(p, q, p^{\prime}, q^{\prime}\right.$ are all prime numbers). The group public key is $\mathcal{Y}=\left(n, a, a_{0}, y=g^{x}, g, h\right)$ where $a, a_{0}, g, h$ are randomly selected $\in_{R} \mathrm{QR}(n)$. The corresponding secret key (known only to $G M)$ is: $\mathcal{S}=\left(p^{\prime}, q^{\prime}, x\right)$.

To join the group, an user engages in a JOIN protocol with the group manager and receives a group certificate $\left[A_{i}, e_{i}\right]$, where $A_{i}=\left(a^{x_{i}} a_{0}\right)^{1 / e_{i}} \bmod n$ with $e_{i} \in \Gamma$ and $x_{i} \in \Lambda$ ( $x_{i}$ is known only to the user).

In order to anonymously sign a message, the group member has to prove possession of his member certificate without revealing it. 
In particular, a group member $P_{i}$ computes:

$$
T_{1}=A_{i} y^{w}, T_{2}=g^{w}, T_{3}=g^{e_{i}} h^{w}, S K(m) .
$$

The value $S K(m)$, over the message $m$, represents a signature of knowledge of (see [ACJT] for details):

1. a value $x_{i}$ such that $\left(a^{x_{i}} a_{0}\right)^{1 / e_{i}}$ is the value that is ElGamal-encrypted in $\left(T_{1}, T_{2}\right)$ under $G M$ 's public key $y$

and

2. an $e_{i}$-th root of that encrypted value, where $e_{i}$ is the first part of the representation of $T_{3}$ w.r.t. $g$ and $h$, such that $e_{i}$ lies in $\Gamma$.

In the event that the signer must be subsequently identified (e.g., in case of a dispute) $G M$ opens the ElGamal encryption to reveal the group certificate $A_{i}$, unique to the member. In addition, $G M$ provides a proof that:

$$
\operatorname{Dlog}_{g} y=\operatorname{Dlog}_{T_{2}}\left(T_{1} / A_{i}\right)
$$

to show that the encryption was opened correctly.

\section{Reissuance-based Revocation in ACJT}

We now discuss two simple revocation schemes for ACJT. When a set of members needs to be revoked, both schemes require the group manager to re-issue group certificates to all remaining members. The first scheme concetrates the cost of reissuance in the group manager while a group member does negligible amount of work. The second scheme distributes the work among the group manager and the idividual members.

\subsection{Scheme I}

Recall that the JOIN protocol in the ACJT scheme results in the issuance of a secret membership certificate $\left[A_{i}, e_{i}\right]$ where $A_{i}=\left(a^{x_{i}} a_{0}\right)^{1 / e_{i}} \bmod n$.

Since the group manager is the one choosing each prime $e_{i}$ at JOIN time, it can easily issue a new certificate to every valid group member without any additional interaction. Specifically, $G M$ can issue a new certificate of the form:

$$
A_{k, i}=\left(a_{k}^{x_{i}} a_{0, k}\right)^{1 / e_{i}} \bmod n, e_{i}
$$

We use the index $k$ to denote the sequence number of $U_{i}$ 's group certificate; equivalently, $k$ is the number of shrinking membership changes that took place since the $U_{i}$ joined the group.

The values $a_{k}$ and $a_{0, k}$ are generated by $G M$ for every update (re-issue) of the group public key. One simple and efficient way of generating these values for $G M$ to select a secret random number $r \in \mathbb{Z}_{p^{\prime} q^{\prime}}^{*}$ and compute $a_{k}=a_{k-1}^{r} \bmod n$ and $a_{0, k}=a_{0, k-1}^{r} \bmod n$. Notice that a revoked user can not obtain a new-issue group certificate since the value $r$ is not known. Furthermore, GM can easily compute the 
value $a_{k}^{x_{i}}$ as $a_{k}^{x_{i}}=\left(a_{k-1}^{x_{i}}\right)^{r}$ (the initial value of $a_{k-1}^{x_{i}}$ can be stored by $G M$ during JOIN).

Next, GM publishes all newly issued certificates in some public forum, e.g., a bulletin board or a web page. Alternatively, it can broadcast the whole batch to the group. Of course, to keep the number of group members secret, $G M$ can (and should) also issue and publish a sufficient number of fake certificates. The new certificates are accompanied by a new group public key:

$$
\mathcal{Y}_{k}=\left(n, a_{k}, a_{0, k}, y, g, h\right)
$$

Obviously, group certificates can not be published in cleartext. Instead, each certificate must be individually encrypted and tagged. One possible format is illustrated in Table 6.1. The purpose of the search tag is to help each member find its new certificate in the table. (Otherwise, a member would have to try decrypting $n / 2$ certificates, on the average, before finding its own.) In this example, every new certificate is encrypted (e.g., using Cramer/Shoup [CS98] under a public key provided by each user at JOIN time). Moreover, a pair-wise secret, $s_{i}$, is established between $G M$ and each group member during JOIN such that the search tag can be computed as, for example, a MAC (a construct such as HMAC-SHA1 would suffice) of a random value $t$ selected at random by the group manager each time the table is re-issued.

\begin{tabular}{|c|c|}
\hline Encrypted Certificate & Search Tag (under $t)$ \\
\hline$E_{1}\left(A_{k, i}, e_{i}\right)$ & $M A C_{s_{1}}(t)$ \\
\hline$\ldots$ & $\ldots$ \\
\hline$\ldots$ & $\ldots$ \\
\hline$\ldots$ & $\ldots$ \\
\hline$E_{n}\left(A_{k, n}, e_{n}\right)$ & $M A C_{s_{n}}(t)$ \\
\hline
\end{tabular}

Table 1. Re-issued Group Certificate Table

The present method is both simple and secure. Unfortunately, it is inefficient, since - for every leaving or expelled member - $G M$ needs to perform $O(n)$ cryptographic operations to compose the table. Moreover, each member needs to fetch the entire certificate table (containing its new certificate) as well as the new group public key. Note that just fetching one's own certificate is insecure as it would reveal to a potential eavesdropper the ownership of a group certificate.

\subsection{Scheme II}

In this section, we propose another simple revocation scheme which off-loads much of the work (required to re-issue a new group certificate) to the individual group members.

Assume the original group public key is $\left(a_{0}, a\right)$, and $n$ members have joined the group with initial group signing certificate $\left(A_{i}, x_{i}, e_{i}\right)$ respectively, obtained when joining the group. Let $f:=e_{1} * \cdots e_{n}$, and assume all members know the value of $f$. We show how to delete a member $k$ as follows. First, GM randomly $u \in Q R_{n}$ as a public 
coin-flipping which ensures that GM cannot determine the value of $u$ as his wish. GM then computes $t:=u^{1 /\left(f / e_{k}\right)} \bmod n$. Then GM publishes a CRL including $t, e_{k}$, and the new public key $\left(a_{0}^{\prime}, a\right)$ where $a_{0}^{\prime}=a_{0} * u$. For a member $i \neq k$, when he sees the CRL, he updates his group signing certificate as $A_{i}^{\prime}=A_{i} * t^{s_{i}}$, where $s_{i}=f /\left(e_{i} * e_{k}\right)$. So the new group signing certificate satisfies $A_{i}^{\prime e_{i}}=a_{0}^{\prime} * a^{x_{i}}$ (note that $a_{0}^{\prime}:=a_{0} * u$ is the new group public key). For member $k$, he cannot compute the corresponding $A_{k}^{\prime}$, so he cannot sign messages corresponding to the new group public key.

We need make a few minor changes to the signing and opening procedure when using this revocation scheme. The group public key $a_{0}^{\prime}$ should be included in hashing in step $2 . b$ in signing procedure in [ACJT]. In the open procedure, assume GM needs to open a signature generated using $\left(A^{\prime}, e\right)$ with the corresponding group public key $\left(a_{0}^{\prime}, a\right)$. GM can compute $A_{i}^{\prime}$ as in the original open procedure, and then check against the signing certificate $\left(A_{i}, e_{i}\right)$ for each member authorized for the corresponding group public key to see whether $A^{\prime}=A_{i} u^{1 / e_{i}}$. If the formula holds, then the signer is member $i$.

This revocation scheme has the following advantages. First, signing and verification operations are constant, i.e. independent on the number of members revoked or in the group. Second, no update is necessary when a new member joins the group. If new members join the group between two revoke events, the information about the newly joined members, namely the $e_{j}^{\prime} s$ of the newly joined members can be accumulated and included in the CRL for the later revoke events. GM only needs to do one exponentiation for each update. When several members are revoked at the same time, it is easy to see that the revocation can be consolidated as one update event. The downside is that when deleting a member, each remaining member needs to do an update that is linear to the number of remaining members.

\section{CRL-based Revocation}

We begin by assuming, as usual, that a CRL is a structure available at all times from a number of well-known public repositories or servers. A CRL is also assumed to be signed and timestamped by its issuer which can be a universally trusted CA, a group manager or some other trusted party.

In addition to the usual components of a group signature scheme (SETUP, JOIN, etc.) we introduce an additional algorithm called REVOKE. Also, as can be expected, revocation influences SIGN and VERIFY algorithms. The JOIN and OPEN components remain unchanged. The only (addition) change in SETUP is as follows:

SETUP (new step) :

- Select $\bar{G}=<\bar{g}>$ of order $n$ in which computing discrete logarithms is hard. For example, $\bar{G}$ can be a subgroup of $Z_{\bar{p}}^{*}$ for a prime $\bar{p}$ such that $n$ divides $(\bar{p}-1)$.

The new REVOKE algorithm shown below is executed by the group manager whenever a member (or a collection of members) leaves or is expelled. (Note that REVOKE may also be executed as a "decoy", i.e., without any new membership revocation activity.) The cost to the $G M$ is linear in the number of revoked group members. 
REVOKE: (We use $s$ to denote the index of the current CRL issue.)

1. First, choose a random element $b_{s} \in_{R} \mathrm{QR}(n)$ (of order $p^{\prime} q^{\prime}$ ). $b_{s}$ becomes the current revocation base.

2. WLOG, assume that $m$ users: $U_{1}, \ldots, U_{m}$ are to be revoked.

3. For each revoked $U_{j}, 1 \leq j \leq m$ compute:

$$
V_{s, j}=b_{s}^{e_{j}}
$$

4. The actual revocation list is then published:

$$
C R L_{s}=b_{s},\left\{V_{s, j} \mid 0<j<m+1\right\}
$$

In the amended SIGN algorithm, as part of step 1, member $U_{i}$ generates two additional values:

$$
\begin{gathered}
T_{4}=f=\bar{g}^{r} \text { where } r \in_{R} Z_{n} \\
T_{5}=f^{b_{s}^{e_{i}}} \bmod n
\end{gathered}
$$

$U_{i}$ then proves, in zero knowledge, that the double discrete logarithm of $T_{5}$ with bases $f$ and $b_{s}$, respectively is the same as the discrete logarithm of $T_{3}$ 's representation base $g$. Since $T_{3}$ is computed as $g^{e_{i}} h^{w}$ mod $n$, the resulting proof of knowledge (SKLOGEQLOGLOG) is verifiable if and only if the same $e_{i}$ is used the construction of both $T_{5}$ and $T_{3}$. The details of this proof are presented below.

Remark: the current CRL value $b_{s}$ must be signed as part of the message "m" which serves as input to the hash function in the actual signature-of-knowledge. This commits the signer to a specific CRL epoch.

In the amended VERIFY algorithm we introduce a new steps 3 and 4:

3. For each $V_{s, j} \in C R L$, check if:

$$
T_{5}==T_{4}^{V_{s, j}} \bmod n
$$

4. Check SKLOGEQLOGLOG, the proof of equality of double discrete logarithm of $T_{5}$ and discrete logarithm of $T_{3}$ 's representation base $g$.

The intuition behind this scheme is straight-forward: if a member $U_{i}$ is revoked, $V_{s, i}$ is published as part of the current group CRL. Thereafter, in order to produce a group signature, $U_{i}$ needs to prove that $\left(b_{s}\right)^{e_{i}}$ does not appear on the CRL which is impossible since $\left(b_{s}\right)^{e_{i}}=V_{s, j}$ for some $j$ if $U_{i}$ is revoked.

We claim that the new scheme provides backward unlinkability because signatures produced by a revoked user prior to revocation in earlier CRL epochs can not be linked to those produced after revocation. Suppose that an adversary is able to link a prerevocation signature to a post-revocation signature. Then, she can only do so with the help of the new values: $T_{4}$ and $T_{5}$. (Otherwise the ACJT scheme is insecure). Since $T_{4}=f$ is chosen at random for each signature, the only way the adversary can link two signatures is using $T_{5}=f^{b_{s}^{e_{i}}}$. However, this is impossible since the respective $b_{s}$ values are different and unrelated for any pair of signatures computed in different CRL epochs. 
To be more specific, we need to consider two cases: linking two signatures from different CRL epochs and linking two signatures from the same CRL epoch. It is easy to see that both are computationally infeasible assuming DDH is hard. In more detail,

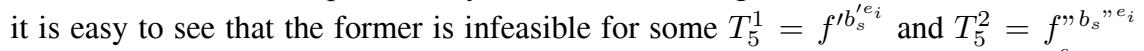
where $f^{\prime} \neq f^{\prime \prime}$ and $b_{s}^{\prime} \neq b_{s}$ ". The latter is also infeasible for some $T_{5}^{1}=f^{\prime b_{s}^{e_{i}}}$ and $T_{5}^{2}=f^{\prime \prime} b_{s}^{e_{i}}$ where $f^{\prime} \neq f^{\prime \prime}$, based on a well-known variant of the DDH problem.

Achieving Unconditional Linkability. The values $T_{6}$ and $T_{7}$ defined above also give the group manager the flexibility of selecting the desired revocation flavor. More specifically, the group manager can decide to revoke all the signatures of a particular member, thus achieving unconditional linkability as defined in Section 4. To do so, it is sufficient to release as part of the CRL the value $e_{i}$, rather than $b_{s}^{e_{i}}$, thus allowing a verifier to check (for any signature) whether $T_{6}^{e_{i}}$ is equal to $T_{7}$.

Obscuring CRL Size. Over time, the size of the CRL may leak some information about the population of the group: by observing long-term changes and fluctuations in the size of the CRL, an adversary can guess the number of group members. For this reason, it may be necessary to obscure the true CRL size. This can be done by introducing a number of fake (but well-formed) CRL entries.

Proofs Involving Double Discrete Logs Proofs of knowledge of double discrete logarithms have been used in the past. Examples include Stadler's technique for publicly verifiable secret sharing [Stad96] and Camenisch/Stadler group signature scheme and its derivatives [CS97,LR98,KP98]. All of the these involve only proofs of knowledge; whereas, in the above scheme, we need a new proof (SKLOGEQLOGLOG) of equality of a double discrete log and a (single) discrete log of a representation. The technique we use is a minor variation of the SKLOGLOG (proof of knowledge of double discrete logarithm) proposed by Camenisch [Cam98], Stadler [Stad96] and Camenisch/Stadler [CS97]. The proof is constructed as follows:

Given $y_{1}=g^{a^{x}}$ and $y_{2}=g_{1}^{x} g_{2}^{w}$, we want to prove that:

$$
\operatorname{Dlog}_{a}\left(\operatorname{Dlog}_{g} y_{1}\right)=\operatorname{Dlog}_{g_{1}}\left(y_{2} / g_{2}^{w}\right)(=x)
$$

Let $\ell \leq k$ be two security parameters and $H:\{0,1\}^{*} \longrightarrow\{0,1,\}^{k}$ be a cryptographic hash function. Generate $2 \ell$ random numbers $r_{1}, \ldots, r_{\ell}$ and $v_{1}, \ldots, v_{\ell}$. Compute, for $1 \leq i \leq \ell, t_{i}=g^{a^{r_{i}}}$ and $t_{i}^{\prime}=g_{1}^{r_{i}} g_{2}^{v_{i}}$. The signature of knowledge on the message $m$ is $\left(c, s_{1}, s_{2}, \ldots, s_{\ell}, s_{1}^{\prime}, s_{2}^{\prime}, \ldots, s_{\ell}^{\prime}\right)$, where:

$$
c=H\left(m\left\|y_{1}\right\| y_{2}\|g\| a\|\| g_{1}\left\|g_{2}\right\| t_{1}\|\cdots\| t_{\ell}\left\|t_{1}^{\prime}\right\| \cdots \| t_{\ell}^{\prime}\right)
$$

and

$$
\begin{gathered}
\text { if } c[i]=0 \text { then } s_{i}=r_{i}, s_{i}^{\prime}=v_{i} ; \\
\text { else } s_{i}=r_{i}-x, s_{i}^{\prime}=v_{i}-w ;
\end{gathered}
$$


To verify the signature it is sufficient to compute:

$$
c^{\prime}=H\left(m\left\|y_{1}\right\| y_{2}\|g\||||| g_{1}\left\|g_{2}\right\| \bar{t}_{1}\|\cdots\| \bar{t}_{\ell}\left\|\bar{t}_{1}^{\prime}\right\| \cdots \| \bar{t}_{\ell}^{\prime}\right)
$$

with

$$
\begin{gathered}
\text { if } c[i]=0 \text { then } \bar{t}_{i}=g^{a^{s_{i}}}, \bar{t}_{i}^{\prime}=g_{1}^{s_{i}} g_{2}^{s_{i}^{\prime}} ; \\
\text { else } \bar{t}_{i}=y_{1}^{a^{s_{i}}}, \bar{t}_{i}^{\prime}=y_{2} g_{1}^{s_{i}} g_{2}^{s_{i}^{\prime}} ;
\end{gathered}
$$

and check whether $c=c^{\prime}$.

\section{Efficiency Considerations}

The new revocation scheme presented in Section 7 is quasi-efficient in that a group signature is of fixed size and a signer performs a constant amount of work in generating a signature. This is, as claimed earlier, an improvement on prior results. However, proofs involving double discrete logs are notoriously expensive. For example, if we assume a hash function $H:\{0,1\}^{*} \longrightarrow\{0,1,\}^{k}$ where $k=160$ bits (as in SHA-1), and we assume that the security parameter $\ell=k$, then each SIGN operation will take approximately 500 exponentiations. The cost of VERIFY is roughly the same. Moreover, with a 1024-bit modulus, a signature can range into hundreds of Kbits. This is clearly not efficient.

Remark: one way to reduce the costs of SIGN and VERIFY and (roughly) halve the number of exponentiations is to ask the signer to release as part of SIGN two additional values: $T_{6}=\hat{g}$ and $T_{7}=\hat{g}^{e_{i}}$ for a randomly chosen $\hat{g} \in Q R_{n}$. The signer would show that $T_{7}$ is correctly formed and then prove the equality of the discrete $\log$ base $\hat{g}$ of $T_{7}$ and the double discrete $\log$ of $T_{5}$ (base $f$ and $b_{s}$, respectively). This proof would be both shorter and less costly then the proof of equality of double discrete $\log$ (of $T_{5}$ ) and discrete $\log$ of representation of $T_{3}$.

Despite the usage of double discrete logarithm proofs and in contrast with Bresson and Stern's scheme [BS2000], the cost of SIGN in our scheme is constant (independent of group size of number of revoked members) and signatures are of a fixed size. Comparing with Song's schemes [Song01], our scheme is more expensive for both SIGN and VERIFY due to the double discrete log proof. One advantage of our scheme is in not using fixed (in length and number) time periods. Consequently, a new revocation list can be issued at any time. Also, we introduce no new cryptographic assumptions. Song's two schemes, however, have the benefit of retroactive public revocability meaning that a member's signatures can be revoked for one or more past time periods. This is a feature that our method does not offer.

The cost of REVOKE in our scheme is linear in the number of revoked members: $G M$ performs one exponentiation for each CRL entry $V_{s, j}$. This is comparable with prior results in both [BS2000] and [Song01] schemes. 


\section{Summary and Future Work}

We presented a new revocation method for group signatures based on the ACJT signature scheme [ACJT]. The new method is more practical than prior art due to fixed-size signatures and constant work by signers. On the other hand, it requires the use of proofsof-knowledge involving double discrete logs which results in hundreds of exponentiations per signature. Consequently, revocation in group signatures remains inefficient while the following issues remain open:

- Shorter CRL: in our method a CRL is proportional to the number of revoked members. An ideal scheme would have a fixed-size or, at least, a shorter CRL (e.g., logarithmic in the number of revoked members).

- More efficient VERIFY: the cost of VERIFY is linear in the number of revoked members. It remains to be seen whether a constant- or sublinear-cost VERIFY can be devised.

- Double discrete log: proofs using double discrete logarithms are inefficient, requiring many exponentiations. For revocation to become truly practical, we need to devise either more efficient double discrete log proofs or different revocation structures that avoid double discrete log proofs altogether.

Very recently, Camenisch and Lysyanskaya [CL02] have proposed a new revocation technique which is an improvement over previous work since the verification phase requires constant work. Their technique, based on dynamic accumulators, is quite general and could be applied to several other protocols other than group signatures.

However, it is not yet a completely satisfactory solution for the following reasons:

- The group public key must change even when users are added, whereas, the original group signature scheme avoids this burden.

- The group manager has to maintain two public (and possibly long) lists of values. One list is linear in the number of members ever added (i.e. current plus revoked) to the group and the second list is linear in the number of revoked users. These lists are not technically part of the public key since the verifier does not need them in order to verify the correctness of a signature.

- The lists above have to be available to all group members so that they can use them to sign. On average, a member has to perform a computation that is linear in the number of membership changes that have taken place since the last time he signed. In the worst case, though, the linearity is with respect to the number of current plus revoked group members, which could make the signature procedure inefficient.

\section{References}

[ACJT] G. Ateniese, J. Camenisch, M. Joye, and G. Tsudik. A Practical \& Provably Secure Coalition-Resistant Group Signature Scheme In Advances in Cryptology CRYPTO '00, Springer-Verlag, 2000.

[CP95] L. Chen and T. P. Pedersen. New group signature schemes. In Advances in Cryptology - EUROCRYPT '94, vol. 950 of LNCS, pp. 171-181, 1995. 
[CS97] J. Camenisch and M. Stadler. Efficient group signature schemes for large groups. In Advances in Cryptology - CRYPTO '97, vol. 1296 of LNCS, pp. 410-424, SpringerVerlag, 1997.

[Stad96] M. Stadler. Publicly Verifiable Secret Sharing, In Advances in Cryptology - EUROCRYPT '96, Springer-Verlag, 1996.

[Cam98] J. Camenisch. Group signature schemes and payment systems based on the discrete logarithm problem. PhD thesis, vol. 2 of ETH Series in Information Security an Cryptography, Hartung-Gorre Verlag, Konstanz, 1998. ISBN 3-89649-286-1.

[CvH91] D. Chaum and E. van Heyst. Group signatures. In Advances in Cryptology - EUROCRYPT '91, vol. 547 of LNCS, pp. 257-265, Springer-Verlag, 1991.

[KP98] J. Kilian and E. Petrank. Identity escrow. In Advances in Cryptology - CRYPTO '98, vol. 1642 of $L N C S$, pp. 169-185, Springer-Verlag, 1998.

[LR98] A. Lysyanskaya and Z. Ramzan. Group blind digital signatures: A scalable solution to electronic cash. In Financial Cryptography (FC'98), vol. 1465 of LNCS, pp. 184-197, Springer-Verlag, 1998.

[CS98] R. Cramer and V. Shoup. A practical public key cryptosystem provably secure against adaptive chosen ciphertext attack. In Advances in Cryptology - CRYPTO '98, vol. 1642 of LNCS, pp. 13-25.

[AT99a] G. Ateniese and G. Tsudik. Group Signatures a' la Carte. In Proceedings of 1999 ACM Symposium on Discrete Algorithms, ACM Press, 1999.

[AT99] G. Ateniese and G. Tsudik. Some open issues and new direction in group signatures. In Proceedings of 1999 Financial Crypto. Springer-Verlag, 1999.

[BS2000] E. Bresson and J. Stern. Efficient Revocation in Group Signatures, In Proceedings of Public Key Cryptography (PKC'2001), Springer-Verlag, 2001.

[Song01] D. Song. Practical Forward-Secure Group Signature Schemes. In Proceedings of 2001 ACM Symposium on Computer and Communication Security. November 2001.

[Cam97] J. Camenisch. Efficient and Generalized Group Signatures. In Advances in Cryptology - EUROCRYPT '97, vol. 1233 of LNCS, pp. 465-479, Springer Verlag, 1997

[CM98a] J. Camenisch and M. Michels. A group signature scheme with improved efficiency. In Advances in Cryptology - ASIACRYPT'98, vol. 1514 of LNCS, pp. 160-174, Springer-Verlag, 1998.

[CL02] J. Camenisch and A. Lysyanskaya. Efficient revocation of anonymous group membership certificates and anonymous credentials. IACR eprint archive n. 2001/113. 